\title{
PROBLEMS OF PARTICLE SIZE ANALYSIS
}

A MEETING under this title was held at Hatfield College of Technology on April 23 in conjunction with an exhibition of particle size measuring equipment. Although the meeting, organized by the Whiting and Industrial Powders Research Council, had been intended as an informal regional gathering, it attracted an attendance of about 200 people.

The chairman of the meeting, Mr. D. C. Soul, director of the Research Council, introduced Dr. H. Heywood, principal of Woolwich Polytechnic. After defining a powder as a collection of particulate matter the particles of which were small in relation to the space in which they moved-which allows a shoal of herrings or the solar system to be regarded under the same aspect as a powder - the speaker dealt with the fundamentals of particle size analysis. It was, he pointed out, important to examine the powder under the conditions in which it would be used in practice, and a distinction must be made between methods suitable for routine control and research. It was greatly to be desired that all or many of these methods should be standardized, preferably under the auspices of the British Standards Institution. Attention was directed to the classification of methods of particle size analysis which had been prepared by a committee of the Society for Analytical Chemistry and published in the March 1963 issue of The Analyst.

The second speaker was Dr. V. T. Crowl of the Paint Research Station. He explained that he was mainly concerned with pigments the particles of which were largely in the sub-micron range. He compared the various methods used most frequently for the purpose, for example, surface area determinations by Brunauer-Emmett-Teller gas adsorption, the Perkin/Elmer method, and gas permeability, the latter recording only about 70 per cent of the surface area of the first. Direct electron microscope measurement, the apparently most foolproof method, seems to involve an operator error by which oversize particles tend to be neglected. Flying-spot counting measured aggregates optically separable as single particles. Each of the other methods mentioned, like sedimentation, centrifugal methods, Coulter counter, etc., had its own advantages and pitfalls. A brief discussion before the tea interval pointed to the problems experienced by the individual speakers, such as the erratic effects of moisture, the difficulties and uncertainties of dispersion, the unusual aggregating tendencies of magnetic particles like iron oxide pigments, etc.

After tea, Mr. J. F. Hinsley, of Edgar Allen and Company, Ltd., pointed to the importance of particle size analysis for controlling the efficiency of industrial operations such as grinding and classifying. After enlarging on the problems to be solved, the speaker discussed the evolution of an equation for relating the fineness of the feed and product of a ballmill to the time of grinding.

Dr. B. H. Kaye, of the Research Council, emphasized the need for not unduly multiplying the analyst's effort. The accuracy of bulk sampling, sample reduction, analysis and data handling must be seen as a whole so that the work at the later stages should not be more complex than is warranted by the results of the earlier stages. He mentioned a number of time-saving devices for routine calculations which he had developed. One problem he had successfully overcome was the mixing effect experienced in centrifugal analysis every time the centrifuge was stopped and started. The solution consisted in introduc- ing the sample while the centrifuge was running steadily. The method was demonstrated with an actual model. The fact that it gave reproducible results sufficiently accurate for routine analysis was confirmed by a number of speakers from the floor during the later discussion.

The remainder of the meeting was devoted to further discussion of problems raised both in the speeches and by visitors. Once again the need for standardizing analytical operations and training operators was emphasized. More effort was required to correlate results obtained by different methods. Several speakers stressed the fact that no method measured actual sizes of irregularly shaped lumps of matter called particles, but rather some property or another which could be more or less closely related to the idealized sizes of such lumps, thus explaining why no two or more methods necessarily gave concordant results. One member of the audience complained about the manufacturer's difficulties when he found that his careful sieving tests, even though reproducible when carried out by himself at the customer's works, was not reproduced by the customer's staff, who therefore suspected the manufacturer's methods. The solution of this problem would seem to require frequent recalibration and scrapping of even the best quality test sieves when worn.

Another speaker mentioned that supposedly standardized glass beads contained an erratic number of tiny air bubbles, so that they were not of uniform specific gravity as required. Once the operator suspects their existence, the bubbles can easily be detected by immersing the beads in a liquid of similar refractive index like nitrobenzene. On the brighter side a reasonable explanation was put forward why, by a compensation of errors, valid results could often be obtained from theoretically objectionable methods. These points are only a selection from among those which were raised.

The three-hour meeting was closed by the chairman of the Research Council, Mr. A. H. Thorneloe, who thanked the speakers and the audience for their contributions. He hoped that the Research Council would organize similar regional discussion groups and that full use would be made of the newly formed Industrial Powders Division by firms concerned with any aspect of powder technology.

The exhibition of equipment for particle size analysis, which had been specially arranged for the meeting, was quite as popular as the meeting itself. It comprised 22 stands showing British and overseas equipment for analysis of particle size, with all main and many subsidiary methods of analysis being represented. Included were sedimentation, optical, and turbidimetric instruments as well as those deriving particle size from specific surface, such as adsorption and permeability apparatus. Test sieves complying with the recently amended British Standard Specification 410 were shown. One of the highlights of the exhibition was the 'research' model of the disk centrifuge earlier demonstrated by Dr. Kaye, which had been lent by the Atomic Energy Authority.

This meeting was only the first of a series which the Research Council intends to organize in various parts of the United Kingdom, not only on the subject of particle size analysis but also on a number of other aspects of powder technology. Arrangements are in hand for a meeting expected to be held in Sheffield on October 2, when the subject will again be some aspect of powder technology. 